\title{
Erratum: Combining spatiotemporal and particle-number degrees of freedom [Phys. Rev. A 98, 043841 (2018)]
}

\author{
Filippus S. Roux ๑
}

(Received 11 December 2019; published 28 January 2020)

DOI: 10.1103/PhysRevA.101.019903

In the above-named paper, we derived the completeness condition for the fixed-spectrum coherent states with the aid of a completeness condition for the fixed-spectrum Fock states, which in turn followed from an assumed generalized completeness condition for the normalized complex functions that parametrize the fixed-spectrum Fock states. It turns out that the latter, which is given in Eq. (19) in the original paper, lacks a normalization constant on the right-hand side, which invalidates the completeness condition for the fixed-spectrum Fock states.

To illustrate the problem, we consider the simplest case [Eq. (16) in the original paper, ignoring the spin indices], given by

$$
\int F\left(\mathbf{k}_{1}\right) F^{*}\left(\mathbf{k}_{2}\right) \mathcal{D}[F]=(2 \pi)^{3} \omega_{1} \delta\left(\mathbf{k}_{1}-\mathbf{k}_{2}\right) .
$$

We multiply both sides by $(2 \pi)^{3} \omega_{1} \delta\left(\mathbf{k}_{1}-\mathbf{k}_{2}\right)$ and integrate over $\mathbf{k}_{1}$ and $\mathbf{k}_{2}$. Thanks to the normalization of the Fourier domain wave function [Eq. (10) in the original paper], the left-hand side becomes

$$
\int\left[\int|F(\mathbf{k})|^{2} d k\right] \mathcal{D}[F]=\int \mathcal{D}[F] .
$$

It produces the volume of the function space, which is expected to be equal to the cardinality of the continuum. The right-hand side, on the other hand, gives

$$
\int \delta(0) d^{3} k_{1} \equiv \Omega
$$

which is associated with the cardinality of countable infinity. [Note that the definition of $\Omega$ here differs by a factor of 2 from the definition given in Eq. (A6) in the original paper.] The two sides cannot be equal unless a normalization constant proportional to the cardinality of the continuum is multiplied on the right-hand side. For the generalized completeness condition, given by Eq. (19) in the original paper, this constant would also depend on $N$-the number of pairs of complex functions in the product. The result is that the sum over the occupation number [Eq. (20) in the original paper] should contain these normalization constants as coefficients, giving different weights to the different $n$-particle projection operators. So, the sum is not equal to the identity operator, which implies that the fixed-spectrum Fock states fail to satisfy a completeness condition. The discussion in Sec. II C in the original paper is to be discarded, as well as the paragraph in the original paper that contains Eqs. (61) up to (63).

Consequently, the completeness condition that was derived for the fixed-spectrum coherent states with the aid of the completeness condition of the fixed-spectrum Fock states [Eq. (35) in the original paper] is incorrect. Here, we obtain the correct expression for the (over)completeness of the fixed-spectrum coherent states in a more direct and simpler way. Consider the operator defined by

$$
\hat{L}=\int\left|\alpha_{0}\right\rangle\left\langle\alpha_{0}\right| \mathcal{D}\left[\alpha_{0}\right],
$$

where $\alpha_{0}$ represents the parameter function of the fixed-spectrum coherent states. It is also the field over which the functional integral is evaluated. For

$$
\alpha_{0}=\frac{1}{\sqrt{2}}\left(q_{0}+i p_{0}\right)
$$

the measure implies

$$
\mathcal{D}\left[\alpha_{0}\right]=\mathcal{D}\left[q_{0}\right] \mathcal{D}\left[p_{0}\right] .
$$

Next, we overlap the operator by different coherent states on either side:

$$
\left\langle\alpha_{1}|\hat{L}| \alpha_{2}\right\rangle=\int\left\langle\alpha_{1} \mid \alpha_{0}\right\rangle\left\langle\alpha_{0} \mid \alpha_{2}\right\rangle \mathcal{D}\left[\alpha_{0}\right]=\int \exp \left(-\frac{1}{2}\left\|\alpha_{1}\right\|^{2}-\frac{1}{2}\left\|\alpha_{2}\right\|^{2}-\left\|\alpha_{0}\right\|^{2}+\left\langle\alpha_{1}, \alpha_{0}\right\rangle+\left\langle\alpha_{0}, \alpha_{2}\right\rangle\right) \mathcal{D}\left[\alpha_{0}\right],
$$


where we used Eq. (27) in the original paper. The functional integral over $\alpha_{0}$ produces

$$
\left\langle\alpha_{1}|\hat{L}| \alpha_{2}\right\rangle=(2 \pi)^{\Omega} \exp \left(-\frac{1}{2}|| \alpha_{1}\left\|^{2}-\frac{1}{2}\right\| \alpha_{2} \|^{2}+\left\langle\alpha_{1}, \alpha_{2}\right\rangle\right)=(2 \pi)^{\Omega}\left\langle\alpha_{1} \mid \alpha_{2}\right\rangle .
$$

Since $\left|\alpha_{1}\right\rangle$ and $\left|\alpha_{2}\right\rangle$ are arbitrary fixed-spectrum coherent states, it follows that

$$
\int\left|\alpha_{0}\right\rangle\left\langle\alpha_{0}\right| \mathcal{D}\left[\alpha_{0}\right]=(2 \pi)^{\Omega} \mathbb{1} .
$$

The completeness condition thus reads

$$
\frac{1}{(2 \pi)^{\Omega}} \int|\alpha\rangle\left\langle\alpha\left|\mathcal{D}[\alpha] \equiv \int\right| \alpha\right\rangle\langle\alpha| \mathcal{D}^{\circ}[\alpha]=\mathbb{1},
$$

where

$$
\mathcal{D}^{\circ}[p] \equiv \frac{1}{(2 \pi)^{\Omega}} \mathcal{D}[p] .
$$

We note that the constant is different from what was obtained in the original paper. The paragraphs in the original paper that contains Eqs. (31) up to (36) should be replaced by the derivation in the current paragraph.

The derivation of the orthogonality conditions of the spatiotemporal quadrature bases in the original paper glossed over the orthogonality constants, which led to some inconsistencies. Here, we repeat the relevant parts of the derivation of the orthogonality condition in a more rigorous way. We start with the overlap between elements of the dual quadrature bases [cf. Eq. (88) in the original paper],

$$
\langle q \mid p\rangle=\frac{V_{0} W_{0}}{2^{\Omega / 2}} \exp \left(\frac{1}{2}\|q\|^{2}+\frac{1}{2}\|p\|^{2}+i q \diamond p\right)
$$

where

$$
q \diamond p \equiv \int q(\mathbf{k}) p(\mathbf{k}) d k
$$

Specific expressions were assumed in the original paper for $V_{0}$ and $W_{0}$ to obtain the desired expression for $\langle q \mid p\rangle$ in Eq. (90) in the original paper. However, we only need to specify their product to be

$$
V_{0} W_{0}=2^{\Omega / 2} \exp \left(-\frac{1}{2}\|q\|^{2}-\frac{1}{2}\|p\|^{2}\right) .
$$

Their $q$ and $p$ dependencies require that

$$
V_{0}=\kappa_{q} \exp \left(-\frac{1}{2}\|q\|^{2}\right), \quad W_{0}=\kappa_{p} \exp \left(-\frac{1}{2}\|p\|^{2}\right),
$$

where $\kappa_{q}$ and $\kappa_{p}$ are constants to be determined. They represent the freedom to redefine the two quadrature bases. Therefore, we can control the constant associated with $\langle q \mid p\rangle$ (by requiring that $\kappa_{q} \kappa_{p}=2^{\Omega / 2}$ ) and the orthogonality constant for $\left\langle q \mid q^{\prime}\right\rangle$ (by choosing $\kappa_{q}$ ), but that fixes the orthogonality constant for $\left\langle p \mid p^{\prime}\right\rangle$.

The overlap $\left\langle q \mid q^{\prime}\right\rangle$, expressed as a limit [cf. Eqs. (92) and (93) in the original paper, with some improvements], reads

$$
\left\langle q \mid q^{\prime}\right\rangle=\kappa_{q}^{2} \lim _{\epsilon \rightarrow 0} \frac{1}{(2 \epsilon)^{\Omega / 2}} \exp \left(-\frac{1}{2 \epsilon}\left\|q-q^{\prime}\right\|^{2}\right) .
$$

It then follows that, unless $\left\|q-q^{\prime}\right\|^{2}=0$, the limit would produce zero. It is tempting to conclude that

$$
\left\langle q \mid q^{\prime}\right\rangle=\Lambda_{q} \delta\left[q-q^{\prime}\right]
$$

where $\Lambda_{q}$ is an unknown orthogonality constant, but to be a Dirac delta functional, the quantity must satisfy the requirement

$$
\int W[q]\left\langle q \mid q^{\prime}\right\rangle \mathcal{D}[q]=\Lambda_{q} W\left[q^{\prime}\right]
$$

where $W[q]$ is an arbitrary functional of $q$. Using the expression in Eq. (16), we have

$$
\begin{aligned}
\int W[q]\left\langle q \mid q^{\prime}\right\rangle \mathcal{D}[q] & =\kappa_{q}^{2} \lim _{\epsilon \rightarrow 0} \frac{1}{(2 \epsilon)^{\Omega / 2}} \int W[q] \exp \left(\frac{-\left\|q-q^{\prime}\right\|^{2}}{2 \epsilon}\right) \mathcal{D}[q] \\
& =\kappa_{q}^{2} \lim _{\epsilon \rightarrow 0} \int W\left[\sqrt{2 \epsilon} q_{0}+q^{\prime}\right] \exp \left(-\left\|q_{0}\right\|^{2}\right) \mathcal{D}\left[q_{0}\right]=\kappa_{q}^{2} \pi^{\Omega / 2} W\left[q^{\prime}\right] .
\end{aligned}
$$

Here, we shift the $q$ variable and then absorb into it the $\epsilon$ factor, which then emerges from the measure, causing it to cancel the $\epsilon$ factor in front. The argument of the functional then becomes independent of the integration field in the limit $\epsilon \rightarrow 0$. In the end, the result has the form required for a Dirac delta functional, with the orthogonality constant given by $\Lambda_{q}=\kappa_{q}^{2} \pi^{\Omega / 2}$. 
Setting $\kappa_{q}=\pi^{-\Omega / 4}$, we get $\Lambda_{q}=1$. A similar calculation for the $p$ basis (using $\mathcal{D}[p]$ and not $\mathcal{D}^{\circ}[p]$ ) gives

$$
\int W[p]\left\langle p \mid p^{\prime}\right\rangle \mathcal{D}[p]=\Lambda_{p} W\left[p^{\prime}\right]=\kappa_{p}^{2} \pi^{\Omega / 2} W\left[p^{\prime}\right] .
$$

The choice of $\kappa_{q}$ and Eq. (14) produces $\kappa_{p}=2^{\Omega / 2} \pi^{\Omega / 4}$ and $\Lambda_{p}=(2 \pi)^{\Omega}$. Hence, the orthogonality conditions for the spatiotemporal quadrature bases read

$$
\left\langle q \mid q^{\prime}\right\rangle=\delta\left[q-q^{\prime}\right], \quad\left\langle p \mid p^{\prime}\right\rangle=(2 \pi)^{\Omega} \delta\left[p-p^{\prime}\right],
$$

and the constants in the definitions of the creation operators of the spatiotemporal quadrature bases become

$$
\hat{a}_{q}^{\dagger}=\pi^{-\Omega / 4} \exp \left(-\frac{1}{2}\|q(\mathbf{k})\|^{2}+\hat{a}_{Q}^{\dagger}-\hat{a}_{R}^{\dagger}\right), \quad \hat{a}_{p}^{\dagger}=2^{\Omega / 2} \pi^{\Omega / 4} \exp \left(-\frac{1}{2}\|p(\mathbf{k})\|^{2}+i \hat{a}_{P}^{\dagger}+\hat{a}_{R}^{\dagger}\right) .
$$

This derivation replaces the last part of Sec. IV in the original paper.

The completeness conditions for the spatiotemporal quadrature bases are affected by the constants obtained in the orthogonality conditions. Moreover, they cannot be based on the use of the completeness condition for fixed-spectrum Fock states. Here, we provide a different proof for these completeness conditions. The expression for the completeness has the form

$$
\hat{B}=\int|q\rangle\langle q| \mathcal{D}[q] .
$$

Following the same approach that we used for the fixed-spectrum coherent states, we overlap $\hat{B}$ on either side with different fixed-spectrum coherent states:

$$
\left\langle\alpha_{1}|\hat{B}| \alpha_{2}\right\rangle=\int\left\langle\alpha_{1} \mid q\right\rangle\left\langle q \mid \alpha_{2}\right\rangle \mathcal{D}[q] .
$$

The overlaps between the coherent states and the quadrature basis elements can be calculated directly from the operators for the quadrature basis elements given in Eq. (22). The result differs from what was obtained in Appendix B in the original paper only in the overall constant. It gives

$$
\left\langle\alpha_{1}|\hat{B}| \alpha_{2}\right\rangle=\pi^{-\Omega / 2} \int \exp \left[-\frac{1}{2}\left\|q-q_{1}\right\|^{2}-\frac{1}{2}\left\|q-q_{2}\right\|^{2}-i p_{1} \diamond\left(q-\frac{1}{2} q_{1}\right)+i p_{2} \diamond\left(q-\frac{1}{2} q_{2}\right)\right] \mathcal{D}[q],
$$

where we used Eq. (27) in the original paper and expressed $\alpha_{1}$ and $\alpha_{2}$ in terms of $q$ 's and p's, as in Eq. (5). The functional integration over $q$ produces a factor $\pi^{\Omega / 2}$, which removes the factor in front. The result, expressed in terms of $\alpha_{1}$ and $\alpha_{2}$, reads

$$
\left\langle\alpha_{1}|\hat{B}| \alpha_{2}\right\rangle=\exp \left(-\frac{1}{2}|| \alpha_{1}\left\|^{2}-\frac{1}{2}\right\| \alpha_{2} \|^{2}+\left\langle\alpha_{1}, \alpha_{2}\right\rangle\right)=\left\langle\alpha_{1} \mid \alpha_{2}\right\rangle
$$

which follows from Eq. (27) in the original paper. Since $\left|\alpha_{1}\right\rangle$ and $\left|\alpha_{2}\right\rangle$ can be any coherent states, we find that $\hat{B} \equiv \mathbb{1}$. A similar result is obtained for the $p$ basis, but with a factor of $(2 \pi)^{\Omega}$. Hence, the spatiotemporal quadrature bases obey the completeness conditions

$$
\int|q\rangle\left\langle q\left|\mathcal{D}[q]=\mathbb{1}, \quad \int\right| p\right\rangle\langle p| \mathcal{D}^{\circ}[p]=\mathbb{1} .
$$

This derivation replaces the derivation in Sec. $\mathrm{V}$ in the original paper.

The conclusions in Sec. VI in the original paper remain unaffected. We obtained complete orthogonal bases for the entire Hilbert space of all quantum optical states in the form of spatiotemporal quadrature bases. 